\title{
Sindrome metabólico en pacientes diabéticos tipo 2 e intolerantes a carbohidratos del EBAIS La Mansión, Nicoya
}

Viviana Alvarado-Soto', Manuel Francisco Jiménez-Navarrete ${ }^{2}$

\begin{abstract}
Objetivo: Analizar la frecuencia y las características clínicas del síndrome metabólico (SM), entre los pacientes diabéticos tipo 2 e intolerantes a carbohidratos en control en el EBAIS La Mansión, del área de salud Nicoya, en un período de un año.
\end{abstract}

Materiales y métodos: estudio retrospectivo de 1 año de duración (septiembre de 2001 a agosto de 2002) de valoración de pacientes diabéticos en control en el EBAIS La Mansión, documentando nombre completo, número de expediente, domicilio, edad, sexo, presencia de diabetes e hipertensión arterial y años de evolución de ambas, índice de masa corporal, obesidad abdominal, triglicéridos, lipoproteína de alta densidad y glicemia (en ayunas). Se utilizó la clasificación del NCEP/ATP III norteamericano para la identificación clínica del SM. Se analizan los expedientes clínicos, tanto del EBAIS como del Hospital de La Anexión. Se mide la circunferencia abdominal, tanto a domicilio, como durante días de citas control por intermedio del ATAP.

Resultados: Se estudiaron 67 pacientes diabéticos, el 59,7\% entre los 40 y 60 años de edad, la mayoría mujeres $(80,6 \%)$, de todos ellos el 68,6\% resultaron con SM. Se estudiaron 9 intolerantes a carbohidratos, todas mujeres, 6 entre los 40 y 60 años de edad, de las cuales 6 resultaron con SM. El 45,7\% de los diabéticos tenían de 0-4 años y el 23,9\% de 5-9 años de evolución de su DM, mientras que en las IC, el 83,3\% tenían de 0-4 años de evolución de su problema. Las asociaciones con HTAfueron un 56,5\% y un $100 \%$ entre diabéticos e IC, respectivamente; con obesidad abdominal un $82,6 \%$ y un $83,3 \%$ entre diabéticos e IC, respectivamente; con hipertrigliceridemia un $60,9 \%$ y un $66,7 \%$, respectivamente, y con HDLC baja un $76,1 \%$ y un $66,1 \%$, respectivamente. La tríada de asociación más frecuente para el diagnóstico del SM, tanto en diabéticos como en IC, lo fue hiperglicemia, hipertensión arterial y obesidad abdominal.

Conclusión: Dos terceras partes de los diabéticos e intolerantes a carbohidratos en control en el EBAIS La Mansión son portadores de síndrome metabólico, y la gran mayoría de ambos grupos posee menos de 5 años de evolución del diagnóstico de hiperglicemia. Lo anterior obliga a utilizar estrategias más agresivas en cuanto a detección, promoción de estilos de vida saludable, prevención de factores de riesgo y control metabólico entre los portadores del SM y sus familiares. Se propone además considerar este síndrome como problema de salud pública en Costa Rica.

Descriptores: diabetes mellitus, síndrome metabólico, atención primaria

Recibido: 23 de enero de 2003

Aceptado: 23 de setiembre de 2003

\footnotetext{
Abreviaturas: DM, diabetes mellitus; SM, síndrome metabólico; IC, intolerantes a carbohidratos; EBAIS, equipo básico de atención integral en salud; HDLC, lipoproteína de alta densidad -por iniciales en inglés-; TGL, triglicéridos; NECP, Programa Nacional de Educación en Colesterol -por iniciales en inglés-; ATPIII, Tratamiento de Hipercolesterolemia en Adultos Panel III -por iniciales en inglés-; IMC, índice de masa corporal; HTA, hipertensión arterial; ATAP, asistente técnico de atención primaria.

${ }^{1}$ Coordinadora EBAIS La Mansión, Área e Salud Nicoya.

${ }^{2}$ Coordinador Programa de Pacientes Crónicos. Hospital de La Anexión, Nicoya.

Correspondencia: Viviana Alvarado Soto. EBAIS La Mansión. Nicoya, Guanacaste. Correo electrónico: vivialvarado@yahoo.com. Manuel Fco. Jiménez Navarrete. Hospital de La Anexión. Nicoya, Guanacaste. Correo electrónico: macypace@ racsa.co.cr
}

El Síndrome Metabólico (SM) o Síndrome de Reaveni está conformado por una serie de anormalidades metabólicas, hemodinámicas y renales, cuyos constituyentes principales son: resistencia a la insulina hiperinsulinemia, obesidad abdominal, hipertensión arterial esencial, anormalidades en el ritmo circadiano de la presión arterial y la frecuencia cardiaca, el síndrome diabético dislipidémico, hipercoagulabilidad, hiperuricemia, patología cardiovascular incrementada y grado de microalbuminuria, todas contribuyentes a un riesgo aumentado de morbimortalidad por enfermedades cardiovas- 
culares, especialmente entre los portadores de diabetes mellitus ${ }^{2,3,4,5}$

La combinación de resistencia a la insulina, dislipidemia, hipertensión y obesidad son los determinantes mayores en DM tipo dos, acumulando en diversas investigaciones hasta el $79 \%$ de la varianza de 10 variables ampliamente estudiadas. Se conoce que hasta el $20 \%$ de las personas no diabéticas portadoras de este "cuarteto mortal", tal y como lo denominó Deedwania, ${ }^{6}$ pueden favorecer la aparición de diabetes mellitus tipo 2 en menos de 5 años ${ }^{7,8,9}$.

En los Estados Unidos de Norteamérica, por ejemplo, la prevalencia del SM aumentó en un $61 \%$ en la última década y la obesidad fue el principal contribuyente a ese aumento. Lo anterior motivó al Colegio Americano de Endocrinología y a la Asociación Americana de Endocrinólogos Clínicos, en agosto de 2002, a declarar esta patología como "epidémica"10. En ese país, la tasa ajustada por edad del SM es del 23,7\%, siendo muy similar en ambos sexos, y se estima que aproximadamente 47 millones de estadounidenses son portadores de este síndrome ${ }^{11}$.

En Costa Rica se documenta solo una investigación del síndrome $\mathrm{X}$ en cien pacientes obesos y diabéticos tipo 2, llevada a cabo en el tercer nivel de atención (Hospital Dr. Calderón Guardia). ${ }^{12}$

Los factores de riesgo cardiovascular múltiples para SM son más comunes en las personas diabéticas que en las no diabéticas. Por ejemplo, el $50 \%$ de los diabéticos son hipertensos, el $50 \%$ son también dislipidémicos y hasta el $80 \%$ son obesos $^{13,14}$.

El objetivo de este estudio es analizar la frecuencia y las características clínicas del SM, entre los pacientes diabéticos e intolerantes a carbohidratos en control en el EBAIS La Mansión, del área de salud Nicoya, en el período de 2001 a 2002.

\section{Materiales y métodos}

Estudio retrospectivo de 1 año de duración (septiembre de 2001 a agosto de 2002). Se valoran los pacientes diabéticos e intolerantes a carbohidratos en control en el EBAIS La Mansión, documentando las siguientes variables: nombre completo, número de expediente, domicilio, edad, sexo, presencia de diabetes o intolerancia a carbohidratos (según clasificación de las guías costarricenses $)^{15}$ e hipertensión arterial y años de evolución de estas, índice de masa corporal, obesidad abdominal, trigliceridemia, lipoproteinemia de alta densidad y glucemia. Todos los parámetros de hematobioquímica fueron determinados con los pacientes en ayunas.

Se utilizó la clasificación del National Cholesterol Education Program (NCEP/AT P III) ${ }^{16}$ para la identificación clínica del SM, basada en cinco factores de riesgo: obesidad abdominal (hombres $>102 \mathrm{~cm}$ y mujeres $>80 \mathrm{~cm}$ ), triglicéridos $(151$ $\mathrm{mg} / \mathrm{dl}$ o más, como cifra anormalmente elevada), lipoproteína de alta densidad (hombres $<40 \mathrm{mg} / \mathrm{dl}$ y mujeres $<50 \mathrm{mg} / \mathrm{dl} \mathrm{co-}$ mo cifras anormales), cifras de presión arterial (130/85 mmHg ó más) y glicemia en ayunas (110 mg/dl o más).

Se analizaron los expedientes clínicos de los pacientes diabéticos, tanto del EBAIS La Mansión como del Hospital de La Anexión. Se midió la circunferencia abdominal, a domicilio y en las citas control, por el ATA P del EBAIS.

Se registraron las variables en una base de datos en el programa Excel de ambiente Window $®$, se recopilaron los datos de los pacientes en una tabla previamente validada con un grupo de pacientes diabéticos hecha en el Hospital, y las variables se procesaron mediante el paquete estadístico SAS System.

\section{Resultados}

Se estudiaron 67 pacientes diabéticos, de los cuales el 80,6\% eran mujeres. El promedio de edad fue de 57,8 años. El $59,7 \%$ del total de pacientes se encontraban entre los 40 y 60 años de edad. El 68,6\% resultaron con síndrome metabólico (Cuadro 1).

\begin{tabular}{|cccccc|}
\hline \multicolumn{6}{|c|}{ Cuadro № 1} \\
Diabéticos y porcentaje de portadores de \\
síndrome metabólico, según grupo etario y sexo. \\
EBAIS La Mansión, área de salud Nicoya, \\
Números absolutos y porcentuales. \\
\hline \multicolumn{5}{|c}{ Hombres } \\
Edad & № & Con SM & № & Con SM & Con SM (\%) \\
\hline $30-40$ & 0 & 0 & 5 & 3 & 4.47 \\
$40-49$ & 2 & 0 & 6 & 4 & 5.97 \\
$50-59$ & 3 & 1 & 18 & 16 & 25.4 \\
$60-60$ & 7 & 5 & 13 & 10 & 22.4 \\
70 o más & 4 & 1 & 9 & 6 & 10.4 \\
Total & 16 & 7 & 51 & 39 & 68.64 \\
\hline
\end{tabular}

Fuente:expedients médicos del EBAIS La Mansión y el Hospital de La Anexión, Nicoya.

\begin{tabular}{|c|c|c|}
\hline \multicolumn{3}{|c|}{$\begin{array}{c}\text { Tabla № } 2 \\
\text { Intolerantes a carbohidratos con síndrome } \\
\text { metabólico según edad. EBAIS } \\
\text { La Mansión de Nicoya, Años 2001-2002 }\end{array}$} \\
\hline \multicolumn{3}{|l|}{ Mujeres } \\
\hline Edad & № & Con SM \\
\hline $30-39$ & 1 & 0 \\
\hline $40-49$ & 4 & 3 \\
\hline $50-59$ & 2 & 1 \\
\hline $60-69$ & 1 & 1 \\
\hline 70 o más & 1 & 1 \\
\hline Total & 9 & 6 \\
\hline \multicolumn{3}{|c|}{$\begin{array}{l}\text { Fuente: expedientes médicos del EBAIS La Mansión y } \\
\text { Hospital de La Anexión, Nicoya. }\end{array}$} \\
\hline
\end{tabular}


Se estudiaron 9 intolerantes a carbohidratos (IC), todas mujeres, 6 entre los 40 y 60 años de edad, 6 de ellas resultaron con SM (Cuadro 2).

El 45,7\% de los diabéticos tenían de 0-4 años de evolución de su DM y el 23,9\%, de 5-9 años de evolución de su DM, mientras que en las IC, el 83,3\% tenían de 0-4 años de evolución de su hiperglucemia (Cuadro 3).

El 43,7\% de los diabéticos asociaban de 5 a 9 años de evolución de su HTA, mientras que el $80 \%$ de los intolerantes a carbohidratos asociaban de 0 a 4 años de evolución.

Contando diabéticos e IC, el 58,8\% de los pacientes estudiados presentaban cifras elevadas de triglicéridos en sangre (promedio $173 \mathrm{mg} / \mathrm{dl}$ ); el 52,9\%, cifras bajas de HDLC.

El promedio de IMC fue $28,9 \mathrm{Kg} / \mathrm{m}^{2}$. Un $36,9 \%$ de todos los pacientes estudiados presentaban IMC $>30$, el 34,2\% entre 25 y 29,9 y el $27,3 \%$ entre 20 y $24,9 \mathrm{Kg}$ por metros cuadrados, respectivamente.

La asociación de hipertensión arterial en los portadores de SM fue del $56,5 \%$ con diabéticos y el $100 \%$ con IC, con obesidad abdominal en el $83 \%$ con diabéticos y en el $83 \%$ con IC, con hipertrigliceridemia el $61 \%$ con diabéticos y el $67 \%$ con IC y con HDLC bajo el $76 \%$ con diabéticos y el $66 \%$ con IC, respectivamente (Cuadro 4).

\section{Discusión}

La importancia de controlar adecuadamente, en las personas portadoras de DM e IC, no solo sus cifras de glucosa sanguínea, sino también sus niveles de presión arterial y lípidos en sangre, es un hecho claramente demostrado en varios estudios de medicina basada en la evidencia, ya elaborados y en vías de publicarse. El SM es una entidad de alta relevancia en salud pública y no un ejercicio teórico de análisis en miles de pacientes alrededor del mundo ${ }^{17}$.

Dos terceras partes de los diabéticos e intolerantes a carbohidratos, en control en el EBAIS La Mansión son portadores de SM; la gran mayoría, adultos jóvenes con pocos años de evolución del desarrollo de hiperglicemia ( 0 a 4 años) y de hipertensión arterial (5 a 9 años).

Este síndrome significa trastornos metabólicos que tempranamente van a predisponer a la aparición de complicaciones en la mayoría de estos pacientes. No debe considerarse este síndrome como parte de las etapas finales de la evolución de la DM o de la hipertensión arterial en poblaciones de adultos mayores.

Los resultados obligan a utilizar en este EBAIS nicoyano, estrategias más agresivas en cuanto a detección, promoción de estilos de vida saludable, prevención de factores de riesgo y control metabólico entre los portadores del síndrome metabólico y sus familiares.

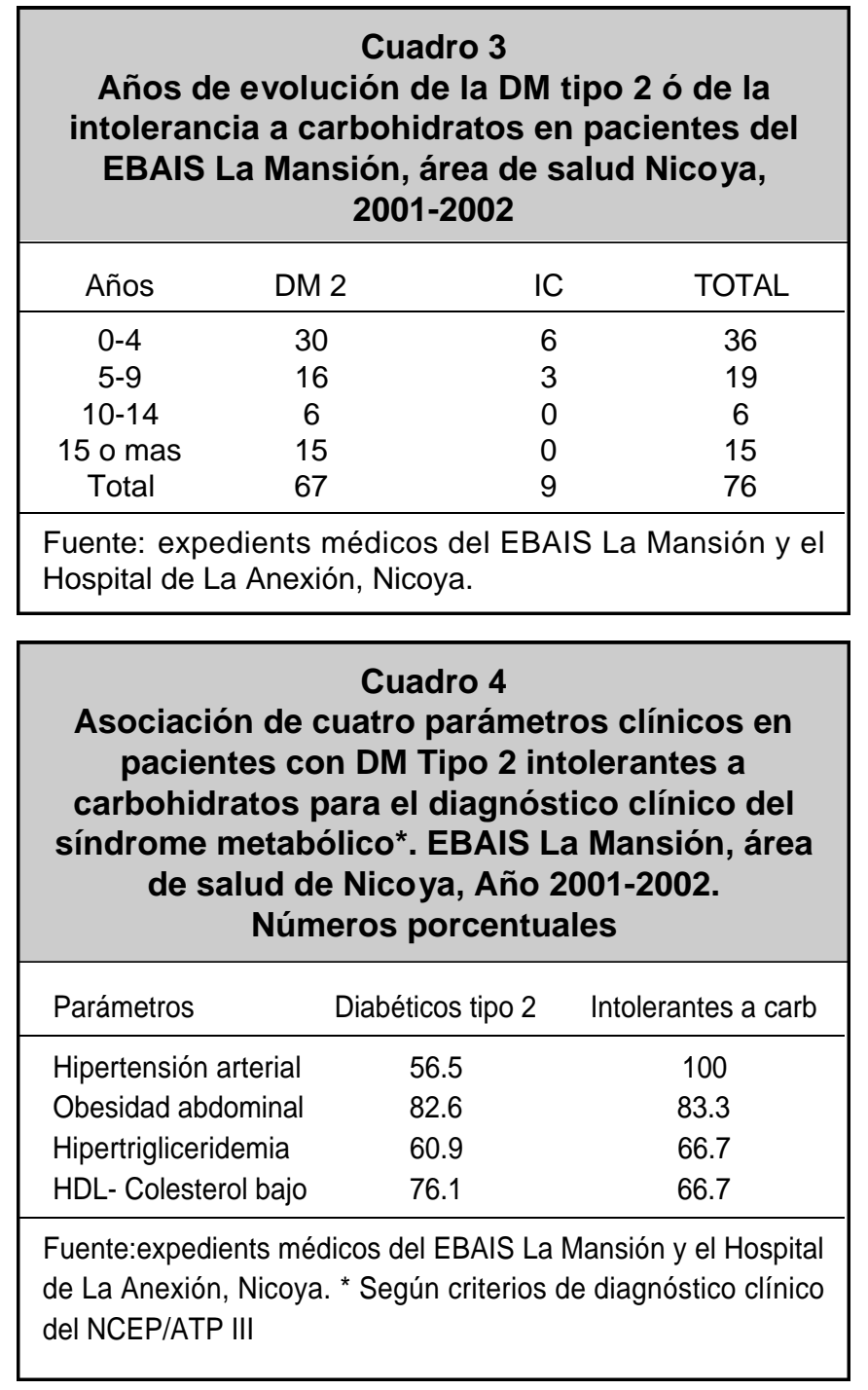

La tríada de asociación más frecuente para el diagnóstico del SM, tanto en diabéticos como en IC, lo fue hiperglucemia, hipertensión arterial y obesidad abdominal. Está bien documentado internacionalmente que la reducción de lípidos y de la presión arterial produce descensos significativos en los eventos coronarios en diabéticos ${ }^{18}$.

El hecho de que alrededor del $70 \%$ de estos pacientes presentaban IMC superiores a 25 (la mitad de ellos superiores a 30), obliga a establecer estrategias de reducción de peso y otras modificaciones inmediatas en estilos de vida, dado el riesgo a corto plazo, de eventos cardiovasculares a los que están expuestos estos pacientes.

Este es el primer informe de SM en una población de pacientes crónicos en el nivel inicial de atención en salud costarricense, y coadyuva a que se le brinde la importancia debida a la detección oportuna y al manejo eficaz de estos pacientes que, por su complejidad fisiopatológica, no pueden ser atendidos enfocando solo diabetes mellitus o hipertensión arterial separadamente, sino con un abordaje eficaz de toda su problemática metabólica. 
Este estudio evidencia una realidad de salud pública, en una zona geográfica distante de los mayores centros poblacionales del país. El SM debería contemplarse como problema de salud pública en las políticas nacionales de atención integral.

El control del portador de síndrome metabólico es muy diferente al paciente que es solamente diabético, hipertenso o inclusive ambos. Se debe conscientizar a los equipos de salud a cargo del manejo de los pacientes crónicos, sobre la necesidad de que esta población se controle más agresiva y estrechamente, con el fin de preservar lo mejor posible su calidad de vida.

\section{Abstract}

In order to determine the presence of metabolic syndrome (MS) between hiperglycemic patients (type 2 diabetics and those with glucose intolerance) at a primary care center ( $\mathrm{La}$ Mansión), a retrospective study was carried out between september 2001 to august 2002. The clinical diagnosis of MS was based on the guidelines of the NCEP/ATPIII. There were 67 diabetic patients, 59,7\% of them between 40 and 60 years old and $80,6 \%$ were women. $68,6 \%$ of the 67 had MS. There were 9 patients with glucose intolerance, all women, 6 with MS. $45,7 \%$ of the diabetics and the $83,3 \%$ of the patients glucose intolerance with MS, have been diagnosed with hiperglycemia between 0-4 years. Associations with hypertension were $56,5 \%$ and $100 \%$ between diabetics and glucose intolerance respectively, with abdominal obesity were $82,6 \%$ and $83,3 \%$ between diabetics and glucose intolerance respectively, with high triglycerides $60,9 \%$ and $66,7 \%$ between diabetic and glucose intolerance respectively, with low highdensity lipoprotein $76,1 \%$ and $66,1 \%$ between diabetics and glucose intolerance respectively. The 3 most frecuent clinical associations of MS were fasting glucose $>110 \mathrm{mg} / \mathrm{dl}$, hypertension and abdominal obesity. Around $60 \%$ of diabetics and glucose intolerance patients clinic had MS and the majority with only few years of the diagnosis of hiperglycemia and hypertension. In this community we need to use aggressive measures in changing lifestyles and metabolic control in these patients and in their families. It is necessary to consider MS as a public health problem in Costa Rica.

\section{Agradecimiento}

Los autores desean expresar el más sincero agradecimiento al Sr. Marcos Reyes Villarreal (asistente técnico de atención primaria del EBAIS La Mansión) y a la Dra. Adriana Vergara (directora del Laboratorio Clínico, Hospital de La Anexión), por la valiosa colaboración brindada.

\section{Referencias}

1. Reaven GM. Syndrome X: 6 years later. J Intern Med 1994; 236 (suppl 736): 13-22.

2. Sowers JR. Update on the Cardiometabolic Syndrome. Clin Cornerstone 2001; 4: 17-23.

3. Reusch JAE. Current concepts in insulin resistance, type 2 diabetes mellitus, and the metabolic syndrome. MdConsult.com 2002, noviembre. PII S0002-9149(02)02555-9.
4. Schafer et al. AWaist is a Terrible Thing to Mind: Central Obesity, the Metabolic Syndrome, and Sleep Apnea Hypopnea Syndrome. MdConsult.com 2002, noviembre. http://www.mdconsult.com/das/journal/view/23771866/

5. Pietropaolo M and Le Roith D. Pathogenesis of Diabetes: Our Current Understanding. Clin Cornerstone 2001; 4: 1-16.

6. Deedwania PC. The Deadly Quartet Revisited. Am J Med 1998; 105 (1A): 1S-3S.

7. Hanson RL, Imperatore G, Bennett PH y Knowler WC. Components of the "Metabolic Syndrome" and Incidence of Type 2 Diabetes. Diabetes 2002; 51: 3120-3127.

8. Hsueh WAy Law RE. Cardiovascular risk continuum: implications of insulin resistance and diabetes. Am J Med 1998; 105 (Suppl 1A): 4S$14 \mathrm{~S}$.

9. Goldstein BJ. Insulin resistance as the core defect in type diabetes mellitus. MdConsult.com 2002, noviembre. PII S0002-9149(02)025535 .

10. Editor. Stalking Syndrome X. En: http;//www.hopkinsafter50.com, diciembre 2002.

11. Ford, ES, Giles WH y Dietz WH. Prevalence of the Metabolic Syndrome Among US Adults. Findings From the Third National Health and Nutrition Examination Survey. JAMA2002; 287: 356-359.

12. Fernández E. YMorera O. Síndrome X en Costa Rica (diabetes mellitus tipo II, obesidad con estigma dérmico). Rev Méd de Costa Rica y CA1997; 538: 35-38.

13. Bohannon NJV. Coronary artery disease and diabetes. Postgraduate Medicine 1999; 105: 66-79.

14. Bosch X, Alfonso F y Bermejo J. Diabetes y enfermedad cardiovascular. Una Mirada hacia la nueva epidemia del siglo XXI. Rev Esp Cardiol 2002; 55: 525.

15. Caja Costarricense de Seguro Social. Dirección Técnica de Servicios de Salud. Manual para la Atención Integral e la Diabetes Tipo 2. En el Primer Nivel de Atención. Unidad de Imprenta CCSS. San José, Costa Rica, año 2002. Página 7.

16. Expert Panel on Detection, Evaluation and Treatment of High Blood Cholesterol in Adults. Executive Summary of the Third Report of the National Cholesterol Education Program (NCEP) Expert Panel on Detection, Evaluation and Treatment of High Blood Cholesterol in Adults (Adult Treatment Panel III). JAMA2001; 285 (19): 2486-2497.

17. Abraira $\mathrm{C}$ y Duckworth W. The Need for Glycemic Trials in Type 2 Diabetes. Clinical Diabetes 2003; 21: 107-111.

18. Huang ES, Meigs JB y Singer DE. The effect of interventions to prevent cardiovascular disease in patients with type 2 diabetes mellitus. Am J Med 2001; 111: 633-642. 